\title{
Case-control Study of the Role of the Gitelman's Syndrome Gene in Essential Hypertension
}

\author{
NORIKO AOI*,**, TOMOHIRO NAKAYAMA*,**, NAOYUKI SATO*, KOTOKO KOSUGE**, \\ AKIRA HAKETA**, MIKANO SATO**** AND MASAYOSHI SOMA*** \\ *Division of Molecular Diagnostics, Department of Advanced Medical Science; Nihon University School of Medicine, Tokyo, Japan \\ **Division of Nephrology, Hypertension and Endocrinology, Department of Medicine; Nihon University School of Medicine, Tokyo, \\ Japan \\ ***Division of General Medicine, Department of Medicine; Nihon University School of Medicine, Tokyo, Japan
}

\begin{abstract}
Background: Gitelman's syndrome is an inherited tubular disorder characterized by sodium wasting, low blood pressure, secondary hyperaldosteronism, metabolic alkalosis, hypokalemia, hypomagnesemia of renal origin, and hypocalciuria. The majority of patients with this syndrome carry inactivating mutations in the SLC12A3 gene encoding the thiazide-sensitive $\mathrm{Na}(+)-\mathrm{Cl}(-)$ cotransporter (NCC) located in the distal convoluted tubule, which is involved in renal sodium reabsorption. This suggests that the SLC12A3 gene is involved in mediation of blood pressure levels. The aim of the present study was to investigate relationships between single nucleotide polymorphisms (SNPs) in the human SLC12A3 gene and essential hypertension (EH) in Japanese. Method: We selected 3 SNPs in the human SLC12A3 gene (T180K, A569V, L849H), and performed a case-control study of $315 \mathrm{EH}$ patients and 305 normotensive (NT) individuals. Results: There was no significant difference in overall distribution of genotypes or alleles of any of the SNPs between the EH and NT groups. Conclusion: We conclude that the causal gene of Gitelman's syndrome is not involved in determining blood pressure levels.
\end{abstract}

Key words: Essential hypertension, Gitelman's syndrome, Thiazide-sensitive $\mathrm{Na}(+)-\mathrm{Cl}(-)$ cotransporter, Single nucleotide polymorphism

(Endocrine Journal 55: 305-310, 2008)

THE thiazide-sensitive $\mathrm{Na}(+)-\mathrm{Cl}(-)$ cotransporter (NCC) of the distal convoluted tubule is believed to be the principal mediator of sodium and chloride reabsorption in this nephron segment, accounting for a significant fraction of net renal sodium reabsorption. Human NCC is a 1021-amino-acid glycoprotein with a general topology similar to that of the $\mathrm{Na}(+)-\mathrm{K}(+)-\mathrm{Cl}(-)$ cotransporter 1 (NKCC1) and $\mathrm{NKCC} 2$, with a central core of 12 transmembrane domains. Its official name is solute carrier family 12 (sodium/chloride transporters), member 3 (SLC12A3). The SLC12A3 gene is located at chromosome 16q13, contains 26 exons interrupted

Received: August 9, 2007

Accepted: February 16, 2008

Correspondence to: Dr. Tomohiro NAKAYAMA, Division of Molecular Diagnostics, Department of Advanced Medical Science, Nihon University School of Medicine, 30-1 Ooyaguchikamimachi, Itabashi-ku, Tokyo 173-8610, Japan by 25 introns, and consists of approximately 4.8 kilobase pairs (kbp) $[1,2]$. This cotransporter is the target of thiazide diuretics used in the treatment of high blood pressure [2]. Loss-of-function mutations in the SLC12A3 gene cause Gitelman's syndrome (GS), which is an autosomal recessive disorder characterized by hypokalemic metabolic alkalosis, hypomagnesemia, hypocalciuria and low blood pressure. In animal models, it has been shown that null mutant $(\mathrm{Ncc}-/-)$ mice exhibit a lower blood pressure than wild-type mice during $\mathrm{Na}+$ restriction [3].

Tago et al. [4] have reported that the frequency of GS mutations in the Japanese general population may be higher than previously suspected. They estimated that the incidence of asymptomatic subjects with heterozygous GS mutations in the Japanese general population was $6.4 \%$, and that the incidence of GS patients was $0.1 \%$, with almost no consanguinity. This frequency is similar to that of patients with familial 
hypercholesterolemia, which is the most frequent genetic trait (1 in 500) caused by mutations of the LDL receptor gene. The study by Tago et al. also showed an association between GS mutations and urine $\mathrm{pH}$, but not an association between GS mutations and blood pressure. Cruz et al. [5] reported that in a large Amish kindred with GS, homozygous mutant family members and younger heterozygous mutant family members had impaired renal salt handling and lower blood pressure than their wild-type relatives.

Essential hypertension (EH) is a complex disorder, and is thought to involve multiple environmental and genetic factors that interact to increase blood pressure [6]. Hypertension is a risk factor for cardiovascular disease (CVD) and stroke, which are the major causes of death in Japan. If human SLC12A3 mutations are significantly associated with decreased blood pressure, such an association should be revealed in case-control studies of EH patients and normotensives (NT). SLC12A3 is thought to be one of the genes involved in determining blood pressure [7].

There have been few case-control studies that have examined the SLC12A3 gene and EH. The aim of the present study was to investigate the association between the SLC12A3 gene and EH using single-nucleotide polymorphisms (SNPs) in this gene.

\section{Materials and Methods}

\section{Subjects}

The subjects comprised 2 groups: the EH group and the NT (control) group. The EH group consisted of 315 patients (mean age, $49.2 \pm 7.4$ years) with $\mathrm{EH}$ diagnosed according to the following criteria: sitting systolic blood pressure (SBP) greater than $160 \mathrm{mmHg}$ and/or diastolic blood pressure (DBP) greater than $100 \mathrm{mmHg}$, on 3 occasions within 2 months after the first medical examination. None of the subjects were receiving anti-hypertensive medication. Subjects diagnosed with secondary hypertension were excluded. The NT group consisted of 305 NT control subjects (mean age, $52.9 \pm 11.1$ years). They were selected from volunteers who had been hospitalized for normal health checkups or who were undergoing a regular company medical checkup. None of the NT subjects had a family history of hypertension, and they all had a SBP of less than $130 \mathrm{mmHg}$ and a DBP of less than
$85 \mathrm{mmHg}$. A family history of hypertension was defined as prior diagnosis of hypertension in grandparents, uncles, aunts, parents or siblings. Histories of alcohol and tobacco use were also recorded. Habitual drinkers were defined as individuals who had $>2$ alcoholic beverages per day 3 times every week. Smokers were defined as current or former smokers, whereas non-smokers were defined as subjects with no history of previous or current smoking. Both the EH patients and the NT control subjects were recruited from the northern part of Tokyo, and informed consent was obtained from each individual according to a protocol approved by the Human Studies Committee of Nihon University. The study was carried out according to the principles of the Declaration of Helsinki [8].

\section{Biochemical analysis}

The blood sampling for plasma renin activity (PRA) was performed after $30 \mathrm{~min}$ of bed rest. The plasma concentration of total cholesterol, HDL-cholesterol and PRA, and the serum concentrations of creatinine and uric acid were measured using standard methods in the Clinical Laboratory Department of Nihon University Hospital.

\section{Genotyping}

Three SNPs (T180K, A569V, and L849H) in the human SLC12A3 gene were selected for analysis, because these SNPs have been reported to be the major mutations (T180K; 47.1\%, L849H; 39.5\%, A569V; $11.8 \%$ ) that make up more than $98 \%$ of all of the SNPs of Gitelman's mutations within the general Japanese population (Fig. 1) [4].

Genotypes were determined using a TaqMan ${ }^{\circledR}$ SNP Genotyping Assays kit and a Custom TaqMan ${ }^{\circledR}$ SNP Genotyping Assays kit obtained from Applied Biosystems (ABI, Foster City, CA, USA), together with TaqMan ${ }^{\circledR}$ PCR. When allele-specific fluorogenic probes hybridize to the template during the polymerase chain reaction (PCR), the $5^{\prime}$ nuclease activity of Taq polymerase can discriminate alleles. Cleavage results in an increased emission of a reporter dye that otherwise is quenched by the dye TAMRA. Each 5' nuclease assay requires 2 unlabeled PCR primers and 2 allele-specific probes. Each probe is labeled with a reporter dye (VIC or FAM) at the 5' end and TAMRA at the 3 ' end. Amplification by PCR was performed using a GeneAmp 


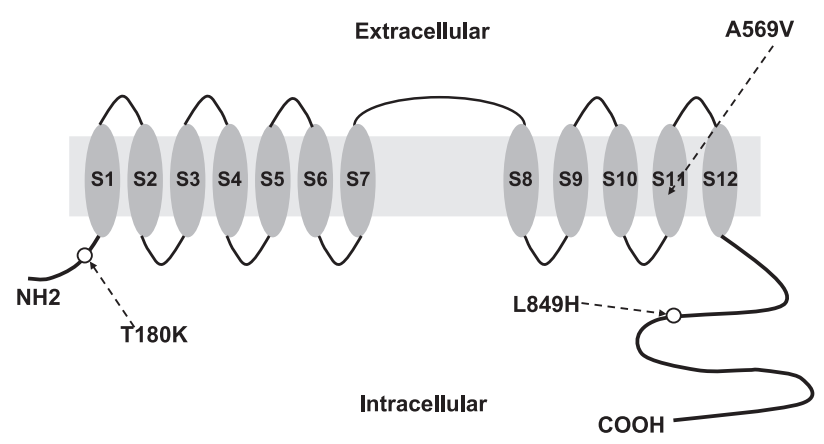

Fig. 1. SLC12A3 mutations. The 3 SLC12A3 SNPs selected are shown in the SLC12A3 scheme. These SNPs are the 3 most frequent SLC12A3 SNPs found in the Japanese population.

9700 system and TaqMan Universal Master Mix (ABI, Branchburg, NJ, USA), with the following thermal cycling protocol: denaturation at $95^{\circ} \mathrm{C}$ for $10 \mathrm{~min}$, followed by 40 cycles of $92^{\circ} \mathrm{C}$ for $15 \mathrm{sec}$ and $60^{\circ} \mathrm{C}$ for $1 \mathrm{~min}$.

Each 96-well plate contained 80 samples of an unknown genotype and 4 reaction mixtures containing reagents but lacking DNA (no-template control). The no-DNA control samples were required for the Sequence Detection System (SDS) 7700 signal processing, as is outlined in the TaqMan Allelic Discrimination Guide (ABI). PCR plates were read using the SDS7700 instrument in the end-point analysis mode of the SDS v1.6.3 software package (ABI). Genotypes were visually determined by comparison with the dyecomponent fluorescent emission data shown in the $\mathrm{X}-\mathrm{Y}$ scatter-plot of the SDS software. Genotypes were also determined automatically by the signal processing algorithms in the software. Results of the 2 scoring methods were saved in separate output files for later comparison [9].

\section{Statistical analysis}

Data are shown as means \pm SD. Hardy-Weinberg equilibrium was assessed by chi-square analysis. Differences in clinical data between the EH patients and NT subjects were assessed using the Mann-Whitney U test. The overall distributions of genotypes and alleles were analyzed using exact tests and multiple logistic regression analysis. Contingency tables including 0 were not assessed. Statistical significance was established at $\mathrm{p}<0.05$.

\section{Results}

Table 1 shows the clinical features of the EH patients and NT controls. Blood pressure, body mass index (BMI), pulse, total cholesterol, uric acid, smoking (\%) and drinking (\%) were significantly higher in the $\mathrm{EH}$ group than in the NT group. Because the mean age of the NT group was greater than that of the EH group, the NT group was regarded as a super-control group [10]. There was no significant difference in the serum concentration of creatinine, plasma concentration of HDL cholesterol or plasma rennin activity between the 2 groups. Table 2 shows the distribution of the genotypes of the 3 SNPs in each group. There was no significant difference in the overall distribution of genotypes of the 3 SNPs between the EH and NT groups. One subject who belonged to the NT group was homozygous for 1 mutation ( $849 \mathrm{H}$ genotype). We also found 1 subject from the EH group who was heterozygous for mutations at 2 different loci $(180 \mathrm{~K}$ and $569 \mathrm{~V}$ genotypes). Table 3 shows the comparison of the clinical data between the subjects with a GS mutation and the subjects without a GS mutation. Between these 2 groups, there was no significant difference for the clinical data.

\section{Discussion}

Activating mutations of the amiloride-sensitive epithelial sodium channel gene cause a type of hypertension known as Liddle's syndrome, whereas inactivating mutations in the same gene cause a type of hypotension known as pseudohypoaldosteronism type 1 [11]. These findings indicate that susceptibility genes of hypertension can also be hypotension susceptibility genes. The aim of the present case-control study was to examine whether the mutant alleles that cause GS are susceptibility variants of EH. If indeed the results show a significant association between these alleles and $\mathrm{EH}$, then this would indicate that these alleles are susceptibility variants of EH. However, the present results showed no significant difference in the distribution of these alleles between the EH and NT subjects, and in addition, there was no difference in the blood pressure between the subjects with the mutant alleles and those without the mutant alleles.

Cruz et al. measured the blood pressure of 199 members of a large Amish kindred with GS [5]. Family 
Table 1. Characteristics of study participants

\begin{tabular}{|c|c|c|c|c|c|c|c|c|c|}
\hline & \multicolumn{2}{|c|}{ Total } & \multirow{2}{*}{$p$ Value } & \multicolumn{2}{|c|}{ Men } & \multirow{2}{*}{$p$ Value } & \multicolumn{2}{|c|}{ Women } & \multirow{2}{*}{$p$ Value } \\
\hline & NT & $\mathrm{EH}$ & & NT & $\mathrm{EH}$ & & NT & $\mathrm{EH}$ & \\
\hline Number of subjects & 305 & 315 & & 196 & 219 & & 109 & 96 & \\
\hline Age (years) & $52.9 \pm 11.1$ & $49.2 \pm 7.4$ & $<0.001$ & $53.2 \pm 8.7$ & $48.3 \pm 8.1$ & $<0.001$ & $52.4 \pm 14.5$ & $51.4 \pm 5.1$ & 0.461 \\
\hline $\operatorname{BMI}\left(\mathrm{kg} / \mathrm{m}^{2}\right)$ & $22.6 \pm 3.2$ & $24.8 \pm 4.0$ & $<0.001$ & $22.8 \pm 3.2$ & $25.1 \pm 3.9$ & $<0.001$ & $22.3 \pm 3.2$ & $24.3 \pm 4.1$ & $<0.001$ \\
\hline $\mathrm{SBP}(\mathrm{mmHg})$ & $112.3 \pm 10.9$ & $173.4 \pm 20.5$ & $<0.001$ & $112.8 \pm 10.3$ & $171.8 \pm 20.2$ & $<0.001$ & $111.4 \pm 11.9$ & $177.0 \pm 21.0$ & $<0.001$ \\
\hline $\mathrm{DBP}(\mathrm{mmHg})$ & $69.1 \pm 8.5$ & $106.3 \pm 14.2$ & $<0.001$ & $69.9 \pm 8.1$ & $107.0 \pm 14.7$ & $<0.001$ & $67.6 \pm 9.2$ & $104.7 \pm 12.9$ & $<0.001$ \\
\hline Pulse (beats/min) & $74.0 \pm 14.5$ & $78.6 \pm 15.3$ & $<0.001$ & $73.4 \pm 15.6$ & $79.1 \pm 15.9$ & $<0.001$ & $75.1 \pm 11.9$ & $77.6 \pm 14.0$ & 0.16 \\
\hline Creatinine (mg/dl) & $0.83 \pm 0.22$ & $0.86 \pm 0.25$ & 0.22 & $0.90 \pm 0.21$ & $0.94 \pm 0.24$ & 0.142 & $0.71 \pm 0.17$ & $0.67 \pm 0.16$ & 0.116 \\
\hline Total cholesterol $(\mathrm{mg} / \mathrm{dl})$ & $200.0 \pm 42.9$ & $210.6 \pm 37.1$ & 0.002 & $195.2 \pm 41.1$ & $207.0 \pm 37.2$ & 0.008 & $209.0 \pm 45.0$ & $218.3 \pm 35.7$ & 0.069 \\
\hline HDL cholesterol $(\mathrm{mg} / \mathrm{dl})$ & $56.2 \pm 17.4$ & $55.4 \pm 17.2$ & 0.459 & $54.1 \pm 16.3$ & $52.0 \pm 16.0$ & 0.16 & $60.1 \pm 18.7$ & $63.1 \pm 17.5$ & 0.291 \\
\hline Uric acid $(\mathrm{mg} / \mathrm{dl})$ & $5.3 \pm 1.5$ & $5.8 \pm 1.6$ & 0.002 & $5.8 \pm 1.4$ & $6.3 \pm 1.5$ & 0.002 & $4.5 \pm 1.2$ & $4.6 \pm 1.2$ & 0.289 \\
\hline $\mathrm{PRA}(\mathrm{ng} / \mathrm{mL} / \mathrm{h})$ & $1.7 \pm 1.8$ & $2.6 \pm 5.9$ & 0.425 & $2.1 \pm 2.1$ & $3.01 \pm 6.8$ & 0.622 & $1.3 \pm 1.3$ & $1.3 \pm 1.8$ & 0.901 \\
\hline Drinking (\%) & 27.6 & 38.5 & 0.001 & 32.6 & 42.8 & 0.008 & 16.2 & 25.6 & 0.068 \\
\hline Smoking (\%) & 20.8 & 33.1 & $<0.001$ & 25.5 & 36.9 & 0.003 & 10.7 & 22.6 & 0.016 \\
\hline
\end{tabular}

All values are means \pm SD. BMI, body mass index; SBP, systolic blood pressure; DBP, diastolic blood pressure; HDL, high density lipoprotein; PRA, plasma rennin activity; NT, normotension; EH, essential hypertension.

Table 2. Genotype Distribution between NT and EH

\begin{tabular}{|c|c|c|c|c|}
\hline \multicolumn{2}{|c|}{ Group (Number of subjects) } & \multirow[t]{2}{*}{ NT (305) } & \multirow[t]{2}{*}{ EH (315) } & \multirow[b]{2}{*}{$p$ value } \\
\hline Variants & Genotype & & & \\
\hline \multirow[t]{6}{*}{$\mathrm{T} 180 \mathrm{~K}$} & $\mathrm{CC}$ & $296(0.970)$ & $310(0.984)$ & \\
\hline & $\mathrm{CA}$ & $9(0.030)$ & $5(0.016)$ & \\
\hline & AA & 0 & 0 & - \\
\hline & Recessive & & & \\
\hline & $\mathrm{AA}+\mathrm{CA}$ & $9(0.030)$ & $5(0.016)$ & \\
\hline & $\mathrm{CC}$ & $296(0.970)$ & $310(0.984)$ & n.s \\
\hline \multirow[t]{6}{*}{ A $569 \mathrm{~V}$} & $\mathrm{CC}$ & $300(0.984)$ & $308(0.978)$ & \\
\hline & $\mathrm{CT}$ & $5(0.016)$ & $7(0.022)$ & \\
\hline & TT & 0 & 0 & - \\
\hline & Recessive & & & \\
\hline & $\mathrm{TT}+\mathrm{CT}$ & $5(0.016)$ & $7(0.022)$ & \\
\hline & $\mathrm{CC}$ & $300(0.984)$ & $308(0.978)$ & n.s \\
\hline \multirow[t]{6}{*}{ L849H } & TT & $297(0.974)$ & $307(0.975)$ & \\
\hline & TA & $7(0.023)$ & $8(0.025)$ & \\
\hline & AA & $1(0.003)$ & 0 & - \\
\hline & Recessive & & & \\
\hline & $\mathrm{AA}+\mathrm{TA}$ & $8(0.026)$ & $8(0.025)$ & \\
\hline & TT & $297(0.974)$ & $307(0.975)$ & n.s \\
\hline
\end{tabular}

The $p$ value of each genotype was calculated using exact test. ns, no significant; NT, normotensive participant; ET, patients with essential hypertension.

members were identified as inheriting $0(n=60), 1$ $(\mathrm{n}=113)$, or $2(\mathrm{n}=26)$ mutations in the SLC12A3 gene. Homozygous mutant family members had significantly lower age- and gender-adjusted systolic and diastolic blood pressures than their wild-type relatives. Heterozygous children, but not adults, had significantly lower blood pressures than their wild-type relatives.
This previous study analyzed family members of GS patients, whereas we conducted a case-control study of EH and NT subjects. Moreover, the SNPs that were selected for analysis differed between the two studies. In another previous study, it was suggested that the distribution of the mutations of the SLC12A3 gene differed between GS patients in Japan and GS patients 
Table 3. GS Mutation and Clinical Data

\begin{tabular}{lcccccc}
\hline & \multicolumn{3}{c}{ NT } & & EH \\
\cline { 2 - 7 } & GS $(+)$ & GS $(-)$ & $p$ value & GS $(+)$ & GS $(-)$ & $p$ value \\
\hline Number of subjects & 22 & 283 & & 19 & 296 & 0.781 \\
BMI $\left(\mathrm{kg} / \mathrm{m}^{2}\right)$ & $23.7 \pm 2.6$ & $22.6 \pm 3.3$ & 0.067 & $26.4 \pm 6.9$ & $24.8 \pm 3.7$ & $173.5 \pm 20.2$ \\
SBP $(\mathrm{mmHg})$ & $114.0 \pm 8.8$ & $112.2 \pm 11.1$ & 0.632 & $171.6 \pm 25.6$ & 0.167 \\
DBP $(\mathrm{mmHg})$ & $68.7 \pm 9.7$ & $69.1 \pm 8.5$ & 0.934 & $107.4 \pm 21.7$ & $106.2 \pm 13.6$ & 0.565 \\
Pulse (beats/min) & $70.6 \pm 11.1$ & $74.2 \pm 14.7$ & 0.283 & $86.8 \pm 19.8$ & $78.0 \pm 14.8$ & 0.057 \\
Creatinine $(\mathrm{mg} / \mathrm{dl})$ & $0.8 \pm 0.3$ & $0.8 \pm 0.2$ & 0.693 & $0.9 \pm 0.2$ & $0.9 \pm 0.3$ & 0.502 \\
Total cholesterol (mg/dl) & $206.2 \pm 54.3$ & $199.5 \pm 42.0$ & 0.564 & $198.3 \pm 28.8$ & $211.3 \pm 37.4$ & 0.206 \\
HDL cholesterol (mg/dl) & $53.5 \pm 17.1$ & $56.4 \pm 17.4$ & 0.744 & $60.0 \pm 23.6$ & $55.2 \pm 16.8$ & 0.568 \\
Uric acid $(\mathrm{mg} / \mathrm{dl})$ & $5.6 \pm 1.8$ & $5.3 \pm 1.5$ & 0.637 & $5.3 \pm 1.7$ & $5.8 \pm 1.6$ & 0.101 \\
PRA (ng/mL/h) & $0.6 \pm 0.6$ & $1.8 \pm 1.8$ & 0.183 & $3.1 \pm 3.6$ & $2.5 \pm 6.0$ & 0.536 \\
\hline
\end{tabular}

GS, Gitelman's syndome; BMI, body mass index; SBP, systolic blood pressure; DBP, diastolic blood pressure; HDL, high density lipoprotein; NT, normotension; EH, essential hypertension; One subject of EH had compound heterozygous genotype (K180 + V569).

in other populations [12].

In our study, 3 SNPs were used for the case-control study. We selected these as functional mutations and not as genetic markers. Some reports have proven the validity of our selection $[13,14]$. A study by Monkawa et al. indicated that the mutations $\mathrm{T} 180 \mathrm{~K}$ and $\mathrm{A} 569 \mathrm{~V}$ of the SLC12A3 gene were inactivating mutations, as the chloride clearance did not increase after thiazide administration in those patients that were homozygous for $\mathrm{T} 180 \mathrm{~K}$ or in a patient that was heterozygous for A569V. Structural analysis has suggested that A569V induces a functional change in the NCC protein structure [13]. Naraba et al. assessed the effects of the SLC12A3 mutation L849H by measuring tracer ${ }^{22} \mathrm{Na}^{+}$ uptake in Chinese hamster ovary cells, and concluded that $\mathrm{L} 849 \mathrm{H}$ is a loss-of-function mutation [14]. Therefore, the 3 SNPs selected for our study were both functional and available for screening for the GS mutation.

In the present study, we found 1 subject from the NT group who was homozygous for L849H. Unfortunately, we were unable to confirm whether this patient had GS, as she was not receiving medication and thus, we were unable to obtain informed consent for drawing and examining her blood.

Additionally, in the present study, we found 1 subject from the EH group who was heterozygous for both T180K and A569V. Again, we were unable to confirm whether that patient had GS, but we suspect that this was not the case, as she did not have hypokalemia or hypomagnesemia.
NCC is responsible for the reabsorption of $5-10 \%$ of the filtered $\mathrm{NaCl}$ [15]. It has also been reported that null mutant (NCC-/-) mice have normal blood pressure, provided that they are not given a low sodium diet [3]. NCC dysfunction may lead to a relatively mild loss of extracellular fluid or sodium loading in the collecting ducts. In the Japanese population, salt intake has been reported to be high versus that seen in other populations [16]. Even though the present study targeted Japanese subjects, any heterozygous mutation that was present might have been insufficient with regard to causing a decrease in the blood pressure in this population.

In summary, we investigated the association between essential hypertension and mutations in the causal gene of GS. The present findings indicate that the GS mutations are not involved in determining blood pressure levels.

\section{Acknowledgments}

We wish to thank Ms. K. Sugama and Mr. A. Morita for their excellent technical assistance. This work was supported by a grant from the Ministry of Education, Culture, Sports, Science and Technology of Japan (High-Tech Research Center, Nihon University), and a research grant from the alumni association of Nihon University School of Medicine. 


\section{References}

1. Mastroianni N, De Fusco M, Zollo M, Arrigo G, Zuffardi O, Bettinelli A, Ballabio A, Casari G (1996) Molecular cloning, expression pattern, and chromosomal localization of the human $\mathrm{Na}-\mathrm{Cl}$ thiazide-sensitive cotransporter (SLC12A3). Genomics 35(3): 486-493.

2. Simon DB, Nelson-Williams C, Bia MJ, Ellison D, Karet FE, Molina AM, Vaara I, Iwata F, Cushner HM, Koolen M, Gainza FJ, Gitleman HJ, Lifton RP (1996) Gitelman's variant of Bartter's syndrome, inherited hypokalaemic alkalosis, is caused by mutations in the thiazide-sensitive Na-Cl cotransporter. Nat Genet 12(1): 24-30.

3. Schultheis PJ, Lorenz JN, Meneton P, Nieman ML, Riddle TM, Flagella M, Duffy JJ, Doetschman T, Miller ML, Shull GE (1998) Phenotype resembling Gitelman's syndrome in mice lacking the apical $\mathrm{Na}^{+}-\mathrm{Cl}^{-}$cotransporter of the distal convoluted tubule. $\mathrm{J} \mathrm{Biol} \mathrm{Chem}$ 273(44): 29150-29155.

4. Tago N, Kokubo Y, Inamoto N, Naraba H, Tomoike H, Iwai N (2004) A high prevalence of Gitelman's syndrome mutations in Japanese. Hypertens Res 27(5): 327-331.

5. Cruz DN, Simon DB, Nelson-Williams C, Farhi A, Finberg K, Burleson L, Gill JR, Lifton RP (2001) Mutations in the $\mathrm{Na}-\mathrm{Cl}$ cotransporter reduce blood pressure in humans. Hypertension 37(6): 1458-1464.

6. Kurtz TW, Spence MA (1993) Genetics of essential hypertension. Am J Med 94(1): 77-84.

7. Lifton RP, Gharavi AG, Geller DS (2001) Molecular mechanisms of human hypertension. Cell 104(4): 545556.

8. Nakayama T, Soma M, Mizutani Y, Xinjuan X, Honye J, Kaneko Y, Rahmutula D, Aoi N, Kosuge K, Saito S, Ozawa Y, Kanmatsuse K, Kokubun S (2002) A novel missense mutation of exon 3 in the type A human natri- uretic peptide receptor gene: possible association with essential hypertension. Hypertens Res 25(3): 395-401.

9. Livak KJ, Marmaro J, Todd JA (1995) Towards fully automated genome-wide polymorphism screening. Nat Genet 9(4): 341-342.

10. Haketa A, Soma M, Nakayama T, Sato M, Kosuge K, Aoi N, Matsumoto K (2004) Two medium-chain acylcoenzyme A synthetase genes, SAH and MACS1, are associated with plasma high-density lipoprotein cholesterol levels, but they are not associated with essential hypertension. J Hypertens 22(10): 1903-1907.

11. Schild L (1996) The ENaC channel as the primary determinant of two human diseases: Liddle syndrome and pseudohypoaldosteronism. Nephrologie 17(7): 395 400.

12. Maki N, Komatsuda A, Wakui H, Ohtani H, Kigawa A, Aiba N, Hamai K, Motegi M, Yamaguchi A, Imai H, Sawada K (2004) Four novel mutations in the thiazidesensitive $\mathrm{Na}-\mathrm{Cl}$ co-transporter gene in Japanese patients with Gitelman's syndrome. Nephrol Dial Transplant 19(7): 1761-1766.

13. Monkawa T, Kurihara I, Kobayashi K, Hayashi M, Saruta T (2000) Novel mutations in thiazide-sensitive $\mathrm{Na}-\mathrm{Cl}$ cotransporter gene of patients with Gitelman's syndrome. J Am Soc Nephrol 11(1): 65-70.

14. Naraba H, Kokubo Y, Tomoike H, Iwai N (2005) Functional confirmation of Gitelman's syndrome mutations in Japanese. Hypertens Res 28(10): 805-809.

15. Obermuller N, Bernstein P, Velazquez H, Reilly R, Moser D, Ellison DH, Bachmann S (1995) Expression of the thiazide-sensitive $\mathrm{Na}-\mathrm{Cl}$ cotransporter in rat and human kidney. Am J Physiol 269: F900-F910.

16. Ministry of Health, Labour and Welfare, Japan (2004) The National Nutrition Survey in Japan, 2002. Tokyo, Dai-ichi Shuppan Press, p. 182. 